\title{
核移植方法和活化方法对小鼠体细胞克隆 胚胎发育的影响
}

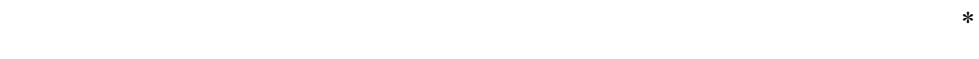

(1) 中国科学院动物研究所计划生育生殖生物学国家重点实验室, 北京 100080; (2) 中国科学院研究生院生物系, 北京 100049 .

*联系人, E-mail: wangliu@,ioz.ac.cn)

摘要 用卵丘细胞为核供体进行小鼠体细胞克隆，成功获得了一批成年体细胞克隆小鼠. 为探讨不同 核移植方法和重构胚活化方法对小鼠克隆胚发育的影响, 用 B6D2F1 小鼠的卵丘细胞和卵母细胞作为 供、受体进行核移植实验, 采用电融合和细胞质内直接显微注射的方法进行核移植, 并采用电脉冲、乙 醇处理、氯化锶处理和电脉冲联合氯化锶 4 种不同方法对重构胚进行活化, 对克隆胚胎的体外及体内发 育进行研究. 结果显示, 利用显微注射法获得重构胚胎的工作效率 $(90.7 \%)$ 显著高于融合法获得重构胚 胎的工作效率(49.7\%). 乙醇、电脉冲和氯化锶 3 种激活方法都可以诱导孤䧳胚发育到囊胚, 但前二者处 理的胚胎的囊胚发育率 $(52.4 \%, 54.2 \%)$ 显著低于后者 $(76.9 \%) .10 \mathrm{mmol} / \mathrm{L}$ 氯化锶处理 $6 \mathrm{~h}$ 活化效果最好. 融合法获得的重构胚经氯化锶或者电脉冲联合氯化锶激活后, 囊胚发育率 $(9.5 \%, 8.6 \%)$ 显著高于单纯电 脉冲激活 $(2.6 \%)$, 而乙醇活化的重构胚没有发育到囊胚阶段. 用显微注射法经氯化锶激活获得的重构胚 的囊胚发育率最高 $(16.6 \%)$. 注射法和融合法获得的重构胚胎经氯化锶激活后进行胚胎移植, 都获得了 发育到期的体细胞克隆小鼠, 发育到期率分别为 $2.5 \%$ 和 $1.4 \%$. 结果表明, 两种核移植方法对 B6D2F1 小鼠重构胚胎的早期发育能力有一定影响, 用这两种方法都可以获得体细胞克隆小鼠; 不同活化方法 对重构胚胎的体外发育能力有显著影响, 在上述 4 种激活方式中, 氯化锶处理对克隆胚的激活效果最 好.

关键词 小鼠 体细胞克隆 核移植 显微操作 卵母细胞激活 发育

核移植是研究细胞核重编程的有力工具. 尽管 目前已获得多种体细胞克隆动物 ${ }^{[1 \sim 9]}$, 但克隆效率仍 然很低. 目前认为导致克隆胚胎、胎儿及克隆动物死 亡率高的重要原因之一是克隆技术不够完善, 包括 核移植方法、胚胎激活方式、胚胎培养及胚胎移植等 技术. Perry报道 ${ }^{[7]}$, 从 1998 年第一只体细胞克隆小鼠 出生以来, 小鼠作为一种优秀的实验动物得到了大 量的应用, 但是小鼠的核移植效率一直很低, 国际上 能够稳定从事小鼠核移植研究的研究组还很少, 这 大大限制了核移植研究的深入.

体细胞克隆小鼠最初是使用压电陶瓷(Piezo)辅 助的直接注射方法获得的 ${ }^{[2]}$, Zhou等人 ${ }^{[10]}$ 使用非 Piezo协助的注射方法同样获得了体细胞克隆后代, 此后大多数克隆小鼠的研究均是采用了 Piezo辅助的 注射技术; 只有少数报道是使用融合方法. Ogura等 人 ${ }^{[11]}$ 使用电融合方法获得尾尖成纤维细胞克隆小鼠. Saito等人 ${ }^{[8]}$ 用融合方法获得胎儿成纤维细胞克隆小
鼠. 同一实验中采用两种常规核移植方法对相同供 体细胞体细胞克隆胚胎发育的影响的研究目前还没 有报道.

卵母细胞的激活是影响克隆效率的重要因素, 因为卵母细胞人工活化方式不能完全模仿受精时卵 细胞的活化 ${ }^{[12]}$, 因而有必要研究各种激活方法对小 鼠体细胞克隆胚的发育的影响, 篮选最优的活化方 法, 提高克隆胚胎发育效率.

为了完善克隆技术，优化小鼠核移植方法和胚 胎激活方式, 本研究使用卵丘细胞作为供体, 比较了 两种核移植方法(电融合方法和直接注射方法)和 4 种 活化方法(电脉冲、乙醇处理、氯化锶处理及电脉冲 组合氯化锶激活)对重构胚发育的影响.

\section{1 材料和方法}

（i ) 试剂. 孕马血精(PMSG)和人线毛膜促性 腺激素(hCG)购自天津市华孚高新生物技术公司，其 
他试剂购自 Sigma 公司.

(ii) 实验动物. DBA 雄鼠和 C57BL/6 倠鼠购自 北京维通利华实验动物中心. B6D2F1 (C57BL/6*DBA) 小鼠为本实验室自行繁殖获得.

(iii) 溶液. 卵母细胞去核前后的培养以及重构胚 的培养用 M16 培养液. 卵母细胞收集及纺㗕体复合体的 去除用 $\mathrm{M} 2$ 液. 电激活液含 $0.05 \mathrm{mmol} / \mathrm{L} \mathrm{CaCl}, 0.1$ $\mathrm{mmol} / \mathrm{L} \mathrm{MgSO}_{4}, 0.3 \mathrm{~mol} / \mathrm{L}$ 甘露醇, $0.1 \mathrm{mg} / \mathrm{mL} \mathrm{PVA,} 3$ $\mathrm{mg} / \mathrm{mL} \mathrm{BSA}$. 融合液含 $0.1 \mathrm{mmol} / \mathrm{L} \mathrm{MgSO}_{4}, 0.3 \mathrm{~mol} / \mathrm{L}$ 甘 露醇, $0.1 \mathrm{mg} / \mathrm{mL}$ PVA, $3 \mathrm{mg} / \mathrm{mL}$ BSA.

(iv) 卵母细胞的收集和供体细胞的准备. 8 16 周龄的 B6D2F1 雌鼠每只腹腔注射 $10 \mathrm{IU}$ 的 PMSG, 48 $\mathrm{h}$ 后腹腔注射 $10 \mathrm{IU}$ hCG, $13 \mathrm{~h}$ 后颈椎离断小鼠并取卵, 获得卵丘卵母细胞复合体后, 放入含 $0.1 \%$ (质量体积 比)的透明质酸酶的 M2 溶液中处理 $3 \mathrm{~min}$, 脱掉卵丘 细胞, 卵母细胞在 M16 溶液中洗 3 遍后放入 M16 溶 液中, 于 $37^{\circ} \mathrm{C}, 5 \% \mathrm{CO}_{2}$ 的空气中培养备用. 卵丘细胞 在 M2 溶液中洗两遍以去除透明质酸酶, 并保存在 M2 液中备用. 使用时挑选直径为 $10 \sim 12 \mu \mathrm{m}$, 圆形, 细胞膜完整的卵丘细胞作为供体.

（v）卵母细胞纺缍体复合体的去除. 卵母细胞纺 缍体复合体的去除(称为去核)及重构操作在 NIKON 显 微镜下进行. 显微操作器为 NIKON-Narishige (MO-188, TOKYO, JAPAN). 卵母细胞放入含 $5 \mu \mathrm{g} / \mathrm{mL}$ 细胞松 驰素 $\mathrm{B}$ 的 M2 液中, 转动卵母细胞使 MII 期染色体中 期板(可见一明亮区域)位于 3 点位置. 去核管的内径 为 $8 \sim 10 \mu \mathrm{m}$ 的平口针, 借助压电陶瓷(Piezo, Prim 1500) 脉冲在 3 点位置的透明带上开一洞, 通过这一洞把去核 平口针抵在明亮区域的纺缍体复合体上, 给一吸力的 时候, 可见复合体堵塞在管口, 然后退针, 纺缍体复合 体即被拉出, 同时仅带出很少量细胞质.

(vi) 核移植方法. (1) 电融合方法. 吸取圆形 的卵丘细胞, 通过去核时所开的洞置于卵周隙内, 并 用针尖按压卵丘细胞, 使其细胞膜与卵母细胞紧密 接触. 重构好的细胞置入融合液中平衡 $1 \mathrm{~min}$, 然后 放入融合室中, 施加 2 次 $2.2 \mathrm{kV} / \mathrm{cm}$ 持续 $10 \mu \mathrm{s}$ 的电 脉冲进行融合, 放入 $\mathrm{M} 16$ 液中, 于 $37^{\circ} \mathrm{C}, 5 \% \mathrm{CO}_{2}$ 的空 气中培养, $30 \mathrm{~min}$ 后在实体显微镜下观察融合情况. (2) 直接注射法. 用内径为 $6 \sim 8 \mu \mathrm{m}$ 的注射针吸取卵 丘细胞, 反复吹出、吸入卵丘细胞使其质膜破裂, 使 细胞核刚好位于注射针管口, 通过去核时透明带上 的洞, 注射针伸入卵胞质内, 然后给一小的 Piezo 脉
冲, 使卵母细胞质膜破开, 注入卵丘细胞核, 退针时 使用负压使卵质膜封闭. 重构好的卵母细胞放入 M16 培养液中培养 1 3 h, 以待活化处理.

(vii) 卵母细胞的活化. 卵母细胞的孤雌活化采 用 3 种方式: (1) $2.2 \mathrm{kV} / \mathrm{cm}$ 持续 $10 \mu \mathrm{s}$ 的两次直流电脉 冲(间隔 $1 \mathrm{~s}$ ); (2) 7\%乙醇的 M16 液处理 $5 \mathrm{~min}$; (3) 氯化 锶处理(含 $10 \mathrm{mmol} / \mathrm{L} \mathrm{SrCl}_{2}$ 和 $5 \mu \mathrm{g} / \mathrm{mL}$ 细胞松驰素 B 的无钙 M16 液) $6 \mathrm{~h}$. 活化处理后, 将电激活组和乙醇 激活组细胞放入含 $5 \mu \mathrm{g} / \mathrm{mL}$ 细胞松驰素 $\mathrm{B}$ 的 M16 液 培养 $6 \mathrm{~h}$. 含有明显原核的细胞记为已激活. 然后将激 活后的卵母细胞放入 $\mathrm{M} 16$ 液中, 于 $37^{\circ} \mathrm{C}, 5 \% \mathrm{CO}_{2}$ 的空 气中培养, 观察体外发育. 对氯化锶活化条件进行了 优化, 使用不同浓度的氯化锶 $(5,10$ 和 $15 \mathrm{mmol} / \mathrm{L})$ 和 不同处理时间(4 和 $6 \mathrm{~h}$ ), 观察孤䧳活化效果.

(viii) 重构胚的活化. 直接注射法获得的重构胚 用含 $10 \mathrm{mmol} / \mathrm{L}$ 氯化锶和 $5 \mu \mathrm{g} / \mathrm{mL}$ 细胞松驰素的无 钲 M16 液处理 $6 \mathrm{~h}$ 进行激活.

电融合方法获得的重构胚用 4 种方法进行激活: (1) $2.2 \mathrm{kV} / \mathrm{cm}$ 持续 $10 \mu \mathrm{s}$ 的两次直流电脉冲; (2) $7 \%$ 乙 醇的 M16 液处理 $5 \mathrm{~min}$ (上述两组细胞处理后, 培养 于含 $5 \mu \mathrm{g} / \mathrm{mL}$ 细胞松驰 B 的 M16 液 $6 \mathrm{~h}$ ); (3) 氯化锶 处理(含 $10 \mathrm{mmol} / \mathrm{L} \mathrm{SrCl}_{2}$ 和 $5 \mu \mathrm{g} / \mathrm{mL}$ 细胞松驰 $\mathrm{B}$ 的无 钙 M16 液) $6 \mathrm{~h}$; (4) $2.2 \mathrm{kV} / \mathrm{cm}$ 持续 $10 \mu \mathrm{s}$ 的两次直流 电脉冲处理, $15 \mathrm{~min}$ 后, 放入含 $10 \mathrm{mmol} / \mathrm{L} \mathrm{SrCl}_{2}$ 和 5 $\mu \mathrm{g} / \mathrm{mL}$ 细胞松驰 B 的无钙 M16 液中 $6 \mathrm{~h}$. 活化处理后, 具有明显类原核结构的胚胎记为已活化胚胎, 培养 于 M16 液中观察体外发育.

(ix) 胚胎移植. 重构胚激活后, 将 1 个细胞克隆 胚移入假孕 $0.5 \mathrm{~d}$ 的昆明白小鼠输卵管膨大部(移植前一 天合笼), 所用的结扎昆明白公鼠在实验前已连续 5 次 交配使母鼠见栓但不育. 于假孕 $19.5 \mathrm{~d}$ 进行剖腹产手术.

(x) 统计分析. 结果用 $\chi^{2}$ 检验分析, $P<0.05$ 记 为差异显著.

\section{2 结果}

\section{1 两种核移植方法获得克隆胚胎的工作效率}

两种方法的重构成功率见表 1. 利用 Piezo 辅助 的直接注射法获得克隆胚胎的效率是 $90.7 \%$ ，显著高 于利用电融合方法获得的克隆胚胎的效率(49.7\%). 经过注射后, $90.7 \%$ 的供体细胞可以成功地导入卵母 细胞胞质内, 而使用电融合方法, 只有近一半的供体 细胞可以成功导入卵母细胞胞质内. 
表 1 不同核移植方法的操作成功率

\begin{tabular}{|c|c|c|c|c|}
\hline \multirow{3}{*}{ 核移植方法 } & \multicolumn{3}{|c|}{ 核移植数(\%) } & \multirow{3}{*}{ 总成功率 $(\%)^{\mathrm{a})}$} \\
\hline & 云核卵数 & 供体导入卵㕣 & 融合成功数 & \\
\hline & & 数(\%) & $(\%)$ & \\
\hline \multirow{2}{*}{$\begin{array}{c}\text { 注射法 } \\
\text { 电融合法 }\end{array}$} & 162 & \multicolumn{2}{|c|}{147 (90.7) } & $147 / 162(90.7)^{\mathrm{a}}$ \\
\hline & 380 & $368(96.8)$ & $189(51.4)$ & $189 / 380(49.7)^{\mathrm{b}}$ \\
\hline
\end{tabular}
析, 下同

\section{2 小鼠卵母细胞的孤䧳发育}

用乙醇、氯化锶以及电脉冲对卵母细胞进行孤雌 活化. 结果表明, 氯化锶激活的孤雌胚胎体外囊胚发 育率 $(76.9 \%)$ 显著高于乙醇和电激活的孤雌胚胎的体 外囊胚发育率(分别为 $52.4 \%$ 和 $54.2 \%$ ), 而乙醇和电 激活间没有显著差异(表 2).

表 2 不同激活方法对孤雌卵体外发育的影响

\begin{tabular}{cccccc}
\hline 激活方法 & 重复数 & 卵母细胞数 & $\begin{array}{c}\text { 活化卵数 } \\
(\%)\end{array}$ & 卵裂数 $(\%)$ & 囊胚数 $(\%)$ \\
\hline $7 \%$ 乙醇 & 3 & 124 & $118(95.2)$ & $105(84.7)$ & $65(52.4)^{\mathrm{b}}$ \\
氯化锶 & 5 & 160 & $154(96.3)$ & $141(88.1)$ & $123(76.9)^{\mathrm{a}}$ \\
电脉冲 & 3 & 118 & $110(93.2)$ & $101(85.6)$ & $64(54.2)^{\mathrm{b}}$ \\
\hline
\end{tabular}

以上结果表明, 氯化锶可有效地激活卵母细胞, 但不同浓度的氯化锶以及处理时间可能影响活化效 果, 本研究进一步比较了 $0,5,10$ 和 $15 \mathrm{mmol} / \mathrm{L}$ 的氯 化锶在不同处理时间(4 和 $6 \mathrm{~h}$ )时的激活能力. 结果表
明, B6D2F1 小鼠在用 $10 \mathrm{mmol} / \mathrm{L}$ 氯化锶处理 $6 \mathrm{~h}$ 激活 效率最高, 囊胚发育率为 $76.9 \%$, 除了相同浓度处理 $4 \mathrm{~h}$ 组外, 显著高于其他浓度及处理时间组(表 3).

\section{3 不同核移植方法和激活方法获得的重构胚的体} 外发育

采用相同的氯化锶活化方法, 注射法获得的重 构胚体外囊胚发育率 $(16.6 \%)$ 显著高于融合法获得的 克隆胚的囊胚率 $(9.5 \%)$ ). 采用融合方法, 氯化锶激活 后囊胚发育率 $(9.5 \%)$ 显著高于电脉冲激活的重构胚 $(2.6 \%)$, 但与电脉冲+氯化锶组合激活组的囊胚发育 率无显著差异. 用乙醇激活的重构胚未发育至囊胚 (表 4).

\section{4 不同核移植方法和激活方法获得的重构胚的体} 内发育

将 1 个细胞重构胚移入假孕 $0.5 \mathrm{~d}$ 昆明白小鼠体 内(结扎公鼠也为昆明白), 于 $19.5 \mathrm{~d}$ 进行剖腹产, 使 用氯化锶激活、注射法重构的胚胎发育到期率为 $2.5 \%$, 用氯化锶激活、融合法重构的胚胎发育到期率 为 $1.4 \%$, 而使用其他激活方式的重构胚没有发育到 期(表 5). 两种重构方法各获得 2 只成活一周以上的 体细胞克隆小鼠.

表 3 不同浓度氯化锶、处理不同时间的孤雌卵体外发育情况

\begin{tabular}{|c|c|c|c|c|c|}
\hline 浓度/mmol· $\mathrm{L}^{-1}$ & 处理时间 $/ \mathrm{h}$ & 卵母细胞数(重复数) & 活化卵数(\%) & 卵裂数(\%) & 囊胚数 $(\%)$ \\
\hline 0 & 6 & $131(6)$ & $0^{\mathrm{c}}$ & $0^{\mathrm{c}}$ & $0^{\mathrm{c}}$ \\
\hline 5 & 6 & $122(3)$ & $98(80.3)^{\mathrm{b}}$ & $90(73.8)^{\mathrm{b}}$ & $48(39.3)^{b}$ \\
\hline 10 & 6 & $160(5)$ & $154(96.3)^{\mathrm{a}}$ & $141(88.1)^{\mathrm{a}}$ & $123(76.9)^{\mathrm{a}}$ \\
\hline 10 & 4 & $158(6)$ & $152(96.2)^{\mathrm{a}}$ & $136(86.1)^{\mathrm{a}}$ & $115(72.8)^{\mathrm{a}}$ \\
\hline 15 & 6 & $120(3)$ & $94(78.3)^{\mathrm{b}}$ & $94(78.3)^{\mathrm{b}}$ & $52(43.3)^{\mathrm{b}}$ \\
\hline
\end{tabular}

表 4 不同核移植方法和激活方法获得的重构胚的体外发育

\begin{tabular}{|c|c|c|c|c|c|c|c|}
\hline \multirow{2}{*}{ 核移植方法 } & \multicolumn{3}{|c|}{ 活化方法 } & \multirow{2}{*}{ 重构胚数 } & \multirow{2}{*}{ 活化胚数 (\%) } & \multirow{2}{*}{ 发育至细胞(\%) } & \multirow{2}{*}{ 发育至囊胚(\%) } \\
\hline & 电脉冲 & 乙醇 & 氯化锶 & & & & \\
\hline 注射 & - & - & + & 162 & $133(82.1)^{\mathrm{a}}$ & $97(59.9)^{\mathrm{a}}$ & $27(16.6)^{\mathrm{a}}$ \\
\hline 融合 & + & - & - & 116 & $91(78.4)^{\mathrm{a}}$ & $61(52.6)^{\mathrm{b}}$ & $3(2.6)^{\mathrm{d}}$ \\
\hline 融合 & - & + & - & 96 & $69(71.9)^{\mathrm{c}}$ & $33(34.4)^{\mathrm{c}}$ & $0(0)^{\mathrm{c}}$ \\
\hline 融合 & + & - & + & 128 & $98(76.6)^{b}$ & $67(52.3)^{\mathrm{b}}$ & $11(8.6)^{b}$ \\
\hline 融合 & - & - & + & 95 & $72(75.8)^{\mathrm{b}}$ & $51(53.7)^{\mathrm{b}}$ & $9(9.5)^{b}$ \\
\hline
\end{tabular}

表 5 不同核移植方法和激活方法获得的重构胚的体内发育

\begin{tabular}{|c|c|c|c|c|c|c|}
\hline \multirow{2}{*}{ 核移植方法 } & \multicolumn{3}{|c|}{ 活化方法 } & \multirow{2}{*}{ 移植肧数 } & \multicolumn{2}{|c|}{ 到期(to term)发育 } \\
\hline & 电脉冲 & 乙醇 & 氯化锶 & & 到期胎儿(\%) & 一周后存活后代(\%) \\
\hline 注射 & - & - & + & 162 & $4(2.5)$ & $2(1.2)$ \\
\hline 融合 & + & - & - & 79 & 0 & 0 \\
\hline 融合 & - & + & - & 92 & 0 & 0 \\
\hline 融合 & + & - & + & 88 & 0 & 0 \\
\hline 融合 & - & nttps: & Sfle & 209 & $3(1.4)^{-}$ & $2(0.96)$ \\
\hline
\end{tabular}




\section{3 讨论}

\section{1 核移植方法与动物克隆}

本研究证明, 用注射法和电融合法均可获得体 细胞克隆小鼠, 且采用两种方法的克隆小鼠发育到 期率都在 $1 \% \sim 3 \%$. 尽管在家畜核移植研究中, 电融 合方法是一种常规的重构方法，但对于小鼠，除了用 4 细胞卵裂球和桑椹胚细胞作供体的核移植外 ${ }^{[13]}$, 电 融合方法很少用于小鼠体细胞核移植研究. 在Ogura 等人 “11的实验中, 所使用的是尾尖成纤维细胞, 其直 径较大, 因而获得了较高的融合率; 卵丘细胞由于直 径小, 融合困难, 因而很少用融合方法进行重构. 本 研究利用融合法获得了 49.7\%的重构成功率, 显著低 于显微注射方法获得的成功率. 尽管如此, 由于显微 注射方法需要较高的操作技能, 电融合方法仍作为 一种重要的核移植方法被采用. 在本研究中, 电融合 获得的重构胚，无论采用何种活化方式，其囊胚发育 率都显著低于显微注射法获得的重构胚，原因可能 在于融合时的电刺激同时对小鼠的卵母细胞具有一 定的活化作用，而此时的卵母细胞对供体核尚未开 始进行重编程, 因而影响了重构胚的体外发育, Wakayama等人江认为延迟活化是他们成功克隆小鼠 的原因之一. 其他的研究者也对核移植所采用的电 融合及注射方法进行了比较. 但对哪种方法更好末 达成一个统一的认识. Galli等人 ${ }^{[14]}$ 报道牛卵母细胞 电融合导入卵丘细胞和成纤维细胞后, 囊胚形成率 显著高于直接注射法, 然而重构胚移入受体牛后受 胎率无显著差异. 相反，Roh等人 ${ }^{[15]}$ 用两种方法核移 植后，猪囊胚形成率无显著差异. Heyman等人 ${ }^{[16]}$ 利 用注射法对牛的细胞进行核移植获得了高的囊胚发 育率. Kawano等人 ${ }^{[17]}$ 认为两种方法对猪的囊胚形成 率、囊胚细胞数、内细胞团与滋养层细胞数都无显著 影响. 这些实验表明, 细胞融合和直接注射方法的核 移植效率可能对动物品种有依赖性. 注射法大大提 高了重构速度, 但其对体内发育的影响仍需进一步 的研究.

\section{2 卵母细胞的孤雌激活}

正常发育中, 哺乳动物卵母细胞由精子激活, 卵 母细胞活化后表现一系列形态变化以及卵胞质内物 质的变化, 包括胞内钻浓度的反复升高、第二极体的 排放、原核形成、DNA的合成和有丝分裂的开始等. M II 期的卵母细胞也可以用人工方式孤䧳激活, 如电
脉冲 ${ }^{[18]}$ 、钙离子载体、氯化锶、乙醇、放线菌酮(一 种蛋白合成抑制剂)，或者这些方式的组合使用 ${ }^{[19,20]}$.

精子入卵引起卵胞质内短暂的周期性的自由钙 浓度的升高, 小鼠及其他哺乳动物这种变化持续数 小时 ${ }^{[21]}$. 受精时这种细胞内游离钻浓度的升高, 可诱 发钻调蛋白激酶 II (CaM II) 的激活, 使 cyclins退化和 CSF灭活, P34 ${ }^{\mathrm{cdc} 2}$ 磷酸化, MPF灭活, 从而使卵子从成 熟分裂阻遏状态释放 ${ }^{[22]}$. 也就是说钙振荡使卵内成 熟促进因子MPF失活，而MPF的作用是使卵子停滞 在 $\mathrm{M}||$ 期 ${ }^{[23]}$. 人工激活卵母细胞的目的在于模仿精 子诱导的卵胞质变化 ${ }^{[24]}$, 锶离子可取代胞内结合态 钙离子, 使钙从胞内钙库释放从而引起自由钙浓度 的升高, 并可引起多次钻升高形成钙振荡 ${ }^{[25]}$. 电脉冲 引起暂时性的膜的穿孔而导致跨膜钙内流，而乙醇 是通过刺激质膜上三磷酸肌醇的形成而引起钙释放 [26]. 本研究中, 使用乙醇、氯化锶、电脉冲对卵母细 胞进行活化处理, 就孤雌激活后原核形成而言, 乙 醇、电脉冲及氯化锶处理之间没有显著差异, 尽管如 此, 氯化锶处理获得了最高的孤雌囊胚发育率. 这是 因为活化后胞质内钻升高的次数、频率、幅度和持续 时间都影响后续的发育, 包括囊胚发育率 $[27]$, 氯化锶 诱导多次胞内钻升高, 乙醇和一次电脉冲只诱导一 次自由钙的升高 ${ }^{[21]}$, 说明重复多次的钙升高对孤䧳 胚胎发育是有利的. 实验证明, 对于孤雌胚胎而言, 在活化过程中，氯化锶的作用时间在 4 和 $6 \mathrm{~h}$ 的时候, 获得的囊胚发育率并没有显著差异. Ma等人 ${ }^{[28]}$ 报道, 在 $10 \mathrm{mmol} / \mathrm{L}$ 氯化锶的浓度下, 作用 $2.5 \mathrm{~h}$ 就可以获得 较高的囊胚发育率. 由此可见, 对于孤雌活化的胚胎 而言, 在 $10 \mathrm{mmol} / \mathrm{L}$ 氯化锶的浓度下, 氯化锶的作用 时间的范围比较大, 并且都可以获得较高的囊胚发 育率.

除了活化试剂的影响, 孤雌活化的效率与取卵 时间 ${ }^{[29]}$ 和小鼠的遗传背景 ${ }^{[30]}$ 也有相关性.

\section{3 不同活化方式激活后重构胚的发育}

在正常受精过程中, 精子进入卵母细胞, 诱发内 质网释放 $\mathrm{Ca}^{2+}$, 使细胞内游离的 $\mathrm{Ca}^{2+}$ 浓度增加, 从而 导致细胞内 MPF 表达水平下降，使停滞在第二次减 数分裂中期的卵母细胞恢复减数分裂, 进入分裂末 期, 形成原核, 使胚胎进入早期发育. 但是在这个过 程中, $\mathrm{Ca}^{2+}$ 浓度的变化是反复振荡的, 会出现数个 $\mathrm{Ca}^{2+}$ 山夆. 㗏胎活化和启动脴胎早期发育的 $\mathrm{Ca}^{2+}$ 浓度并 
不相同, 启动胚胎早期发育的 $\mathrm{Ca}^{2+}$ 浓度要比胚胎活 化所需 $\mathrm{Ca}^{2+}$ 浓度大许多. 因此, 在采用乙醇和电活化 过程中, 这种不充分的活化尽管可以使停滞在第二 次减数分裂中期的克隆胚胎恢复减数分裂, 进入分 裂末期, 形成类原核, 但是并不能很好地启动克隆胚 胎早期的发育, 因此胚胎早期囊胚发育率显著降低.

本研究使用不同的激活方式处理重构胚时, 氯 化锶处理获得的囊胚发育率显著高于乙醇和电激活. 本研究还同时使用了电脉冲和氯化锶的组合方式进 行激活, 希望这种方式能更好地模仿受精时的情形, 因为受精时的首次钙升高的幅度要高于后续的升高 幅度 ${ }^{[31]}$, 但是这种组合方式没有提高克隆胚的囊胚 发育率. 这可能仍然是因为它们引起的钙变化次数 的不同造成的, 氯化锶引起的多次钲峰更加类似于 受精时发生的情况; 另一方面可能在于其引起钙变 化的细节不同造成的. 在正常受精中, 首次钙升高的 幅度大, 持续时间长, 而且是叠加的, 随后的钙振荡 频率低 ${ }^{[31]}$ ，而且在第一次有丝分裂的原核核膜破裂 时, 又出现了钲振荡 ${ }^{[32]}$, 而这些在用孤雌激活方法时 没有实现. 在孤䧳活化中, 几种激活方式都可以获得 较高的囊胚发育率, 但在克隆胚中的情形不同, 因而 克隆胚可能比孤雌胚需要更精细的模仿受精时的钙 变化模式. 有研究表明, 在兔子的核移植中, 间断性 的提供电脉冲不仅可以显著增加激活率和融合率, 而且更重要的是重构胚的发育率也有了明显的提高 [33]. 但也有研究表明, 电融合后一段时间的培养对于 供体核的重编程(reprogramming)是重要的, 并且在 不同种属的动物中, 所需要重编程的时间是不一样 的 29$]$.

锶离子是一种 2 价阳离子, 是一种钙离子的替代 物. 在活化过程中, 锶可以诱导内质网的内源钙离子 释放, 用氯化锶激活, 无论是注射法, 还是融合法, 在进行了重构胚移植后都获得了发育到期的后代, 而使用其他激活方式未获得到期发育. 在另一篇报 道中, 即使使用精子提取物激活重构胚, 发育到期率 与氯化锶激活没有显著差异 ${ }^{[34]}$. 这可能是因为精子 因子引起的钙振荡的频率要显著地高于正常受精时 的钙振荡 ${ }^{[35]}$. 所有这些表明, 克隆胚的活化机制有其 特殊性, 特别是首次钙升高的模式, 以及有丝分裂过 程中的钙振荡, 因而充分了解克隆胚的活化机制, 优 化活化方式有望进一步提高克隆效率.

作为影响克隆效率的主要因素之一, s核移植和
活化方法是研究的热点, 也在不断改进中. Kishigami等 人还报道，在活化过程中加入甲基化药物Trichostatin $\mathrm{A}$, 可以显著提高体外囊胚率、发育到期率和分离干细 胞的效率.

本研究结果表明, 使用卵丘细胞作供体, 电融合 和注射方法都可以获得体细胞克隆小鼠，但克隆效 率仍然在 $1 \% \sim 3 \%$, 因而操作技术可能不是引起克隆 胚发育率低的根本原因. 电脉冲和乙醇处理都可以 有效地诱导孤雌胚发育至囊胚, 但诱导克隆胚的囊 胚发育能力大大下降. 能引起多次钙振荡的氯化锶 处理对克隆胚的激活效果最好.

\section{参考文献}

1 Wilmut I, Schinieke A E, McWhir J, et al. Viable offspring derived from fetal and adult mammalian cells. Nature, 1997, 385: 810813 [DOI]

2 Wakayama T, Perry A C F, Zuccotti M, et al. Full-term development of mice from enucleated oocytes injected with cumulus cell nuclei. Nature, 1998, 394: 369-374[DOI]

3 Polejaeva I A, Chen S H, Vaught T D, et al. Cloned pigs produced by nuclear transfer from adult somatic cells. Nature, 2000, 407: 86-90[DOI]

4 Chesne P, Adenot P G, Viglietta C, et al. Cloned rabbits produced by nuclear transfer from adult somatic cells. Nat Biotechnol, 2002, 20: 366-369[DOI]

5 Zhou Q, Renard J P, Friec G L, et al. Generation of fertile cloned rats by regulating oocyte activation. Science, 2003, 302: 1179[DOI]

6 Lee B C, Lim M K, Jang G, et al. Dogs cloned from adult somatic cells. Nature, 2005, 436(7051): 641[DOI]

7 Perry A C. Nuclear transfer cloning and the United Nations. Nat Biotechnol, 2004, 22(12): 1506-1508[DOI]

8 Saito M, Saga A, Matsuoka H. Production of a cloned mouse by nuclear transfer from a fetal fibroblast cell of a mouse closed colony strain. Exp Anim, 2004, 53(5): 467-469[DOI]

9 Woods G L, White K L, Vanderwall D K et al. A mule cloned from fetal cells by nuclear transfer. Science, 2003, 301(5636): 1063[DOI]

10 Zhou Q, Laurent B, Renard J P. A simplified method for the reconstruction of fully competent mouse zygotes from adult somatic donor nuclei. Cloning, 2000, 2: 35-44[DOI]

11 Ogura $\mathrm{A}$, Inoue $\mathrm{K}$, Takano $\mathrm{K}$, et al. Birth of mice after nuclear transfer by electrofusion using tail tip cells. Mol Reprod Dev, 2000, 57(1): 55-59[DOI]

12 Alberio R, Zakhartchenko V, Motlik J, et al. Mammalian oocyte activation: Lessons from the sperm and implications for nuclear transfer. Int J Dev Biol, 2001, 45(7): 797-809

13 Tsunoda Y, Kato Y. Full-term development after transfer of nuclei from 4-cell and compacted morula stage embryos to enucleated oocytes in the mouse. J Exp Zool, 1997, 278: 250-254[DOI]

14 Galli C, Lagutina I, Vassiliev I, et al. Comparison of microinjection (piezo-electric) and eell fusion for nuclear transfer success with differ- 
ent cell types in cattle. Clon Stem Cells, 2002, 4(3): 189-196[DOI]

15 Roh S, Lee E S, Lee B C, et al. In vitro development of porcine nuclear transfer embryos reconstructed by microinjection of somatic cell nuclei into oocytes using Piezo-driven micromanipulator. Theriogenology, 2001, 55: 288(abstact)

16 Heyman Y, Zhou Q, Lebourhis D, et al. Novel approaches and hurdles to somatic cloning in cattle. Clon Stem Cells, 2002, 4(1): $47-55[\mathrm{DOI}]$

17 Kawano K, Kato Y, Tsunoda Y. Comparison of in vitro development of porcine nuclear-transferred oocytes receiving fetal somatic cells by injection and fusion methods. Clon Stem Cells, 2004, 6(2): 67-72[DOI]

18 Ozil J P. The parthenogenetic development of rabbit oocytes after repetitive pulsatile electrical stimulation. Development, 1990, 109: $117-127$

19 Hagemann L J, Hillery-Weinhold F L, Leibfried Rutledge M L, et al. Activation of murine oocytes with $\mathrm{Ca}^{2+}$ ionophore and cycloheximide. J Exp Zool, 1995, 271(1): 57-61[DOI]

20 Wang W H, Machaty Z, Ruddock N, et al. Activation of porcine oocytes with calcium ionophore: Effects of extracellular calcium. Mol Reprod Dev, 1999, 53(1): 99-107[DOI]

21 Vitullo A D, Ozil J P. Repetitive calcium stimuli drive meiotic resumption and pronuclear development during mouse oocyte activation. Dev Biol, 1992, 151: 128-136[DOI]

22 Machaty Z, Prather R S. Strategies for activating nuclear transfer embryos. Reprod Fertil Dev, 1998, 10: 599-613[DOI]

23 Dupont G. Link between fertilization-induced $\mathrm{Ca}^{2+}$ oscillations and relief from metaphase II arrest in mammalian eggs: A model based on calmodulin-dependent kinase ॥ activation. Biophys Chem, 1998, 72: 153-167[DOI]

24 Nakada K, Mizuno J. Intracellular calcium responses in bovine oocytes induced by spermatozoa and by reagents. Theriogenology, 1998, 50: 269-282[DOI]

25 Cuthbertson K S R, Whittingham D G, Cobbold P H. Free $\mathrm{Ca}^{2+}$ increases in exponential phases during mouse oocyte activation. Nature, 1981, 294: 754-757[DOI]
26 Loi P, Ledda S, Fulka Jr J, et al. Development of parthenogenetic and cloned ovine embryos: Effect of activation protocols. Biol Reprod, 1998, 58: 1177-1187[DOI]

27 Ikumi S, Asada M, Sawai K, et al. Effect of activation methods for bovine oocytes after intracytoplasmic injection. J Reprod Dev, 2003, 49: 37-43[DOI]

28 Ma S F, Liu X Y, Miao D Q, et al. Parthenogenetic activation of mouse oocytes by strontium chloride: A search for the best conditions. Theriogenology, 2005, 64: 1142-1157[DOI]

29 Koo D B, Kang Y K, Choi Y H, et al. In vitro development of reconstructed porcine oocytes after somatic cell nuclear transfer. Biol Reprod, 2000, 63(4): 986-992[DOI]

30 Ibanez E, Albertini D F, Overstrom E W. Effect of genetic background and activating stimulus on the timing of meiotic cell cycle progression in parthenogenetically activated mouse oocytes. Reproduction, 2005, 129(1): 27-38[DOI]

31 Jones K T, Nixon V L. Sperm-induced $\mathrm{Ca}^{2+}$ oscillations in mouse oocytes and eggs can be mimicked by photolysis of caged inositol 1,4,5-trisphosphate: Evidence to support a continuous low level production of inositol 1,4,5-trisphosphate during mammalian fertilization. Dev Biol, 2000, 225: 1-12[DOI]

32 Bos-Mikich A, Whittingham D G, Jones K T. Meiotic and mitotic $\mathrm{Ca}^{2+}$ oscillations affect cell composition in resulting blastocysts. Dev Biol, 1997, 182(1): 172-179[DOI]

33 Collas P, Robl J M. Factors affecting the efficiency of nuclear transplantation in the rabbit embryo. Biol Reprod, 1992, 46: 492$500[\mathrm{DOI}]$

34 Kishikawa H, Wakayama T, Yanagimachi R. Comparison of oocyte-activating agents for mouse cloning. Cloning, 1999, 1: 153-159[DOI]

$35 \mathrm{Wu} \mathrm{H}$, Smyth J, Luzzi V, et al. Sperm factor induces intracellular free calcium oscillations by stimulating the phosphoinositide pathway. Biol Reprod, 2001, 64(5): 1338-1349[DOI]

36 Kishigami S, Mizutani E, Ohta $\mathrm{H}$ et al. Significant improvement of mouse cloning technique by treatment with trichostatin A after somatic nuclear transfer. Biochem Biophys Res Commun, 2006, 340(1): $183-189$ 